\title{
Adaptive Interleaving for OFDM in TDD systems
}

\author{
S.W. Lei, Vincent K.N. Lau and T.S. Ng \\ swlei@eee.hku.hk, knlau@eee.hku.hk, tsng@eee.hku.hk \\ Department of Electrical \& Electronic Engineering, \\ University of Hong Kong, Pokfulam Road, Hong Kong \\ Tel: (852)-28578483, Fax: (852)-25598738
}

25 July 1999

\begin{abstract}
We proposed a novel interleaving technique, namely adaptive interleaving, which can break the bursty channel errors more effectively than traditional block interleaving. This new scheme resequences the transmitted symbols adaptively according to the instantaneous subcarrier channel state information. In this way, the bursty errors introduced by the channel can be broken more effectively, thus a better BER performance can be achieved. This technique is well-suited to OFDM systems because the CSI values of the whole frame could be estimated at once when transmitted symbols are framed in the frequency dimension. Computer simulation shows that significant SNR gains can be achieved, compared with traditional block interleaving.
\end{abstract}

\section{Introduction}

Orthogonal frequency division multiplexing (OFDM) is a promising technique to deliver high data rate multimedia services over the mobile radio channel [1], [2], [3]. The performance of the high-bit-rate transmission is limited by the severe intersymbol interference (ISI) caused by the frequency selective fading of the dispersive channel. In OFDM, the information to be transmitted is split over a large number of subcarriers and transmitted in parallel, thus increasing the symbol duration. In this way, each subcarrier will experience only flat fading and the ISI can be avoided by inserting a guard interval which is greater than the delay spread, between OFDM symbols.

On the other hand, error correction codes are usually applied to protect digital source data across the hostile mobile wireless channel. Most of the error correction codes are designed to correct random channel errors [4]. However, channel errors caused by the mobile wireless channel are bursty in nature. Hence, interleaving is usually employed to randomize the bursty channel errors so that error correction codes could be more effective. Traditionally, block interleaving is employed and is shown to be effective in many circumstances.

In this paper, we propose a novel interleaving technique called the adaptive interleaving. This scheme could bette: exploit the instantaneous channel variation by rearranging the transmitted symbols according to the channel state in. formation (CSI) of the frame. The objective is to break up long burst of bad CSI sequence so that the overall bit error probability is reduced or minimized. The proposed adaptive interleaving is well-suited for OFDM systems. This is because the transmitted symbols are framed in the frequency dimension and therefore, the CSI values for the whole frame can be estimated at the same instant.

The system is analyzed by extensive simulation and we found that there is a significant SNR gain achieved with this scheme relative to traditional block interleaving in many situations. This paper is organized as follows. The system model is presented in section 2 . In section 3 , we discuss the adaptive interleaving. Simulation results will be presented and discussed in section 4 . Finally, we conclude the paper with a brief summary of results.

\section{System Model}

The system model is shown in figure 1. The input bit streams are first divided into frames and then convolutionally coded. The number of coded bits in a frame is the same as the number of subcarriers. The coded bits are BPSK modulated. The BPSK symbols are then interleaved and modulated by inverse Fast Fourier Transform (IFFT). After adding cyclic prefix, the OFDM symbols are then transmitted through a multipath Rayleigh fading channel with $N_{d}$ discrete paths of equal power. The channel gain is normalized to unity.

The received OFDM symbols are then demodulated by using FFT and the demodulated symbols are given by

$$
y_{m}=H_{m} x_{m}+n_{m}
$$

where $x_{m}$ is the BPSK symbol conveyed by the $m$ th subcarrier, $H_{m}$ the corresponding complex frequency gain, and $n$ is the complex white gaussian noise, of variance $N_{0} / E_{s}$. After deinterleaving, the received symbols are decoded with the Viterbi decoder. With the knowledge of the channel state information, maximum likelihood decoding [5] is achieved with the decision metrics given by

$$
\left|y_{m}-H_{m} x_{m}\right|^{2}
$$

For simplicity, perfect channel state information is assumed. 


\section{Adaptive Interleaving}

The purpose of interleaving is to randomize the bursty channel errors. This can greatly improve the performance of the error-correcting codes, which are often good at correcting single error. In general, interleaving rearranges the order of symbols to be transmitted according to a given rule. At the receiver, the reverse rule is used to restore the original permutation.

Generally, interleaving can be described by a $n \times n$ square matrix with only 1 s and 0 s entry, where $n$ is the number of the symbols input to the interleaver at a time. If the input symbols row vector is $\mathbf{a}$, then the interleaved one will be

$$
\mathbf{a}^{\prime}=\mathbf{a} M
$$

Consider an example of 9 symbols input to a simple $3 \times 3$ block interleaver. The interleaving matrix is

$$
M=\left[\begin{array}{lllllllll}
1 & 0 & 0 & 0 & 0 & 0 & 0 & 0 & 0 \\
0 & 0 & 0 & 1 & 0 & 0 & 0 & 0 & 0 \\
0 & 0 & 0 & 0 & 0 & 0 & 1 & 0 & 0 \\
0 & 1 & 0 & 0 & 0 & 0 & 0 & 0 & 0 \\
0 & 0 & 0 & 0 & 1 & 0 & 0 & 0 & 0 \\
0 & 0 & 0 & 0 & 0 & 0 & 0 & 1 & 0 \\
0 & 0 & 1 & 0 & 0 & 0 & 0 & 0 & 0 \\
0 & 0 & 0 & 0 & 0 & 1 & 0 & 0 & 0 \\
0 & 0 & 0 & 0 & 0 & 0 & 0 & 0 & 1
\end{array}\right]
$$

Note that any interleaving matrix has one and only one 1 in each column and each row. Thus, if the input to the interleaver is a sequence of 1-9 in ascending order, then the output will be

$$
[1,4,7,2,5,8,3,6,9]
$$

$M$ can be written more compactly as $n$ ordered pairs $\{(1,1),(4,2),(7,3), \ldots,(9,9)\}$, which indicates the positions of $1 \mathrm{~s}$ in the matrix. $(i, j)$ means the $j$ th column of the $i$ th row. In other words, $(i, j)$ also means that the $i$ th symbols will be put in the $j$ th position after interleaving.

Similarly, deinterleaving is the same process which permutates the symbols so that the original sequence is restored. Thus the deinterleaving matrix $D$ is just the inverse of $M$. The original sequence is

$$
\mathbf{a}=\mathbf{a}^{\prime} D=\mathbf{a}^{\prime} M^{-1}=\mathbf{a}^{\prime} M^{T}
$$

where $M^{T}$ denotes the transpose of $M$. The deinterleaving matrix, $D$, can be easily derived from $\mathrm{M}$ by reversing each ordered pair $(i, j)$ in $\mathrm{M}$ to $(j, i)$, i.e. $D=$ $\{(1,1),(2,4),(3,7), \ldots,(9,9)\}$.

Traditionally, symbols to be transmitted are grouped into frames in time dimension and we could only estimate the channel state associated with a symbol once at a time. Lacking the CSI for the whole frame, simple block interleaving is often used.

However, in OFDM systems, symbols to be transmitted are grouped into frames in frequency dimension. CSI for the whole frame could be estimated at the same time. With the availability of such information, a more intelligent interleaving scheme can be done.
The idea of adaptive interleaving can be illustrated in figure 2. For illustration, the channel state is simply divided into two states, "Good" and "Bad". "Good" corresponds to a low attenuation while "Bad" corresponds to high attenuation or deep fade. Also, it is assumed that half of the channel states considered are "Good" and half of them "Bad".

Since channel states are correlated in both time and frequency dimension, consecutive of Good or Bad is expected in the trellis if no interleaivng is employed. This is undesirable because Viterbi algorithm choose between a correct path and an error path by the accumulated decision metrics covered by the error path. Since, the minimum Hamming distance of an error path is $D_{\min }$, a consecutive of $D_{\min }$ or more of "Bad" states will make the decoder more likely to eliminate the correct path, thus contributing an error. Therefore, the criteria is to break the bursts of "Bad" channel states along the error path so as to reduce the error event probability.

After block interleaving, "Bad" and "Good" states become more random and there are less consecutive "Bad" states. Thus a better BER performance compared with nointerleaving can be expected. However, as shown in the figure, there are still patterns like "BB" covered by the error paths.

Actually, if the instantaneous channel state sequence is available, a better interleaving scheme is possible. The state could be permutated alternatively with "Bad" and "Good" states, as in figure 2. In this way, bursts of "Bad" channel states are completely eliminated from the error path. Therefore, performance gain in error probability could be achieved, with this adaptive interleaving scheme, compared with block interleaving.

In this paper, we design the adaptive interleaver as follows.

For $k=1,2, \ldots, N_{f}$, let the channel state of the $k$ th subcarrier be $H_{k}$ and the symbols to be transmitted be $a_{k}$ (before interleaving). We first sort the symbols by using the absolute value of the channel state sequence $\left[\left|H_{k}\right|\right]_{k=1}^{N_{f}}$ as index. After sorting, the symbols become, say, $\left[a_{k_{1}}, a_{k_{2}}, \ldots, a_{k_{N_{f}}}\right]$ and the magnitude of the associated channel state become in ascending order, $\left[\left|H_{k_{1}}\right| \leq\left|H_{k_{2}}\right| \leq \ldots \leq\left|H_{k_{N_{f}}}\right|\right]$. The sorted symbols $a_{k_{i}}$ are put into a $4 \times\left(N_{f} / 4\right)$ matrix as follows. Note that the second and the fourth rows are reversed in order.

$$
\left[\begin{array}{llll}
a_{k_{1}} & a_{k_{2}} & \ldots & a_{k_{N_{f} / 4}} \\
a_{k_{N_{f} / 2}} & a_{k_{N_{f} / 2-1}} & \ldots & a_{k_{N_{f} / 4+1}} \\
a_{k_{N_{f} / 2+1}} & a_{k_{N_{f} / 2+2}} & \ldots & a_{k_{3 N_{f} / 4}} \\
a_{k_{N_{f}}} & a_{k_{N_{f}-1}} & \ldots & a_{k_{3 N_{f} / 4+1}}
\end{array}\right]
$$

The symbols are then taken out column by column to form a sequence

$$
\left[a_{p_{1}}, a_{p_{2}}, \ldots, a_{p_{N_{f}}}\right]
$$

The interleaving matrix for the adaptive interleaver is then given by (in compact form)

$$
I=\left\{\left(p_{1}, 1\right),\left(p_{2}, 2\right), \ldots,\left(p_{N_{f}}, N_{f}\right)\right\}
$$

where $p_{i}$ is the index of the $i$ th element in the sequence in (3) and $\left(p_{1}, p_{2}, \ldots, p_{N_{f}}\right)$ is a permutation of the numbers $1,2, \ldots, N_{f}$. 
This permutation of the transmitted symbols will result in a more random mix of "Good" and "Bad" states after deinterleaving. ${ }^{1}$ Therefore, a gain in performance is expected.

At the receiver, the same channel state vector is available. Therefore, the identical interleaving sequence as in (3) could be generated and the the deinterleaving matrix for the adaptive deinterleaver is thus (in compact form)

$$
D=\left\{\left(1, p_{1}\right),\left(2, p_{2}\right), \ldots,\left(N_{f}, p_{N_{f}}\right)\right\}
$$

\subsection{CSI at transmitter}

In order to perform adaptive interleaving, the transmitter has to know the channel state information (CSI) for the current frame. The channel state could be estimated at the receiver with the aid of pilot symbols [6]. Pilot symbols are inserted as shown in figure 3(b) and the CSI of symbols in between pilots are obtained by interpolation.

To avoid massive feedback of CSI from the receiver to the transmitter, we assumed a time division duplexing (TDD) system. TDD is a duplexing method in which both the forward and reverse links share the same radio frequency at different time period, as shown in figure 3(a). Assuming slow fading, the CSI values of the uplink and downlink are highly correlated. For example, the CSI could be estimated at $A$ during the first time slot (when $A$ receives). The estimater CSI is used for deinterleaving and maximum likelihood decoding in the current frame. This estimated CSI is also used for adaptive interleaving at the next frame duration (when A transmits). Assuming slow fading, the CSI value does not change significantly over several frame duration. Hence, massive feedback of CSI is avoided and both the transmitter and the receiver could interleave and de-interleave frames based on the same pattern (because the interleaving pattern is derived from the CSI values).

\section{Simulation Results and Discussion}

Computer simulations were performed to examine the perfor mance of the adaptive interleaving techniques on a multipath Rayleigh fading channel. Results are obtained by varying three parameters, $N_{d}$ the number of discrete multipath in the channel models, $N_{f}$ the frame size (ie. the number of subcarriers in the OFDM system), and $D_{\min }$ the minimum Hamming distance of the convolutional.

The convolutional code used is a $1 / 2$-rate code with three different constraint length $K$ :

$$
\begin{aligned}
& \text { 1. } K=3, g=(5,7) \text { with } D_{\min }=5 \\
& \text { 2. } K=5, g=(23,35) \text { with } D_{\min }=7 \\
& \text { 3. } K=7, g=(133,171) \text { with } D_{\min }=10
\end{aligned}
$$

\footnotetext{
${ }^{1}$ Note that the channel state sequence $\left\{H_{p_{i}}\right\}$ in the trellis will then become $\{B, B, G, G, B, B, \ldots, G, G\}$. As in the discussion above, the channel states are simply divided into "Good" (above median of channe states) and "Bad" (below median of channel states). We design the adaptive de/interleaver to generate $\{B, B, G, G, \ldots, G, G\}$ rather than $\{B, G, B, G, \ldots, B, G\}$ because simulation shows that the former one results in better performance than the latter one.
}

where $g$ is the code generator in octal representation.

Figure 4 shows the probability of bit error against the signal energy to noise ratio for $D_{\min }=5$ and $N_{f}=1024$. At BER of $10^{-5}$, the adaptive interleaving scheme obtain SNR gain of about $4.5 \mathrm{~dB}$ and $5.5 \mathrm{~dB}$ over the traditional block interleaving for $N_{d}=3$ and 9 respectively. When $N_{f}=4096$, an even larger gain ( $13 \mathrm{~dB}$ for $N_{f}=3,12 \mathrm{~dB}$ for $N_{f}=9$ ) can be obtained, as shown in figure 5 .

The BER against SNR graph for $D_{\min }=7$ is shown in figure 6 and figure 7 . When $N_{f}=1024$, We can see that only moderate gain of $1.8 \mathrm{~dB}$ and $1.3 \mathrm{~dB}$ is obtained for $N_{d}=3$ and 9. The gains become larger when $N_{f}=4096$. They are respectively $9 \mathrm{~dB}$ and $6.5 \mathrm{~dB}$ for $N_{d}=3$ and 9 . Computer simulation has also been done for $D_{\min }=10$ and results show that only $0.4-1.2 \mathrm{~dB}$ for $N_{f}=1024$ and $2.7-3 \mathrm{~dB}$ for $N_{f}=4096$ can be obtained.

The performance gain of adaptive interleaving over traditional block interleaving can be summarized in the following table.

\begin{tabular}{cccc}
\hline \hline & $D_{\min }=5$ & $D_{\min }=7$ & $D_{\min }=10$ \\
\hline \multicolumn{4}{c}{$N_{f}=1024$} \\
$N_{d}=3$ & $5.5 \mathrm{~dB}$ & $1.8 \mathrm{~dB}$ & $1.2 \mathrm{~dB}$ \\
$N_{d}=6$ & $5.3 \mathrm{~dB}$ & $1.5 \mathrm{~dB}$ & $0.6 \mathrm{~dB}$ \\
$N_{d}=9$ & $4.5 \mathrm{~dB}$ & $1.3 \mathrm{~dB}$ & $0.4 \mathrm{~dB}$ \\
\hline \multicolumn{4}{c}{$N_{f}=4096$} \\
$N_{d}=3$ & $13 \mathrm{~dB}$ & $9 \mathrm{~dB}$ & $2.8 \mathrm{~dB}$ \\
$N_{\mathrm{d}}=6$ & $12.5 \mathrm{~dB}$ & $8 \mathrm{~dB}$ & $2.7 \mathrm{~dB}$ \\
$N_{d}=9$ & $12 \mathrm{~dB}$ & $6.5 \mathrm{~dB}$ & $3 \mathrm{~dB}$ \\
\hline \hline
\end{tabular}

Several observations can be made. Firstly, the performance gain decreases quickly as $D_{\min }$ increases and it becomes insignificant when $D_{\min }=10$. This is because for large $D_{\min }$, the block interleaving could produce a relatively random mix of "Good" and "Bad" states. Therefore, the marginal gain by adaptive interleaving will decrease as $D_{\min }$ increases.

Secondly, we can also see that for a given $N_{f}$, the performance of both the block and adaptive interleaving increases with $N_{d}$. This is simply because increasing $N_{d}$ is equivalent to reducing the coherence bandwidth of channel. This leads to larger order of diversity in frequency dimension. However, since the performances of both interleaving methods are increasing, it is difficult to observe a trend in the gain variation.

Thirdly, for a given $N_{d}$ and when $N_{f}$ becomes large, the performance of block interleaver degrades, while that of adaptive interleaver remains relatively stable. Therefore, this results in a larger SNR gain.

\section{Conclusion}

This paper illustrates a new interleaving scheme, namely adaptive interleaving. This scheme could break long burst of channel error more effectively than traditional block interleaving and is well suited for OFDM systems. This is because in OFDM, transmitted symbols are framed in the frequency dimension and hence, the CSI values of the frame could be estimated at the same instant. The transmitted symbols are 
adaptively resequenced according to the instantaneous channel states of the whole frame.

It has been found that significant performance gain (up to $13 \mathrm{~dB}$ ) can be achieved with the new scheme. The following trends on gain variation are observed too, where $N_{f}$ is the frame size and $N_{d}$ is the number of discrete multipath of the channel (which depends on the coherence bandwidth of the environment).

\section{1. $N_{f}$ fixed}

when $N_{d}$ increases, both block and adaptive interleaving improve in performance. Thus, it is difficult to see the trend on gain variation.

2. $N_{d}$ fixed

performance of block interleaving degrades while that of adaptive interleaving remain relatively stable, when $N_{f}$ increases. Therefore, gain increases with $N_{f}$.

\section{References}

[1] B. L. Floch, M. Alard, and C. Berrou, "Coded Orthogonal Frequency Division Multiplex," Proc. IEEE, vol. 83, pp. 982-996, Jun 1995.

[2] W. Y. Zou and Y. Wu, "COFDM: an overview," IEEE Transactions on Broadcasting, vol. 41, pp. 1-8, Mar 1995.

[3] M. Okada, S. Hara, and N. Morinaga, "Bit Error Rate Performances of Orthogonal Multicarrier Modulation Radio Transmission Systems," IEICE Trans. on Communs., vol. E76-B, pp. 113-119, Feb 1993.

[4] J. Costello, D.J., J. Hagenauer, H. Imai, and S. Wicker, "Applications of error-control coding," IEEE Transactions on Information Theory, vol. 44, pp. 2531-2560, Oct 1998.

[5] D. Divsalar and M. K. Simon, "Trellis Coded Modulation for 4800-9600 bits/s Transmission Over a Fading Mobile Satellite Channel," IEEE J. Selected Areas in Communications, vol. SAC-5, pp. 162-175, Feb 1987.

[6] Y. Li, L. J. C. Jr., and N. R. Sollenberger, "Robust Channel Estimation for OFDM Systems with Rapid Dispersive Fading Channels," IEEE Trans. on Communs., vol. 46, pp. 902-915, Jul 1998.

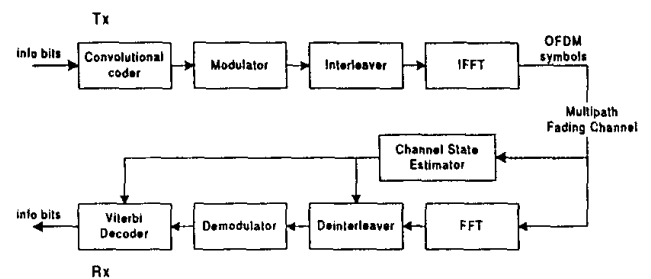

Figure 1: Block diagram of coded OFDM system

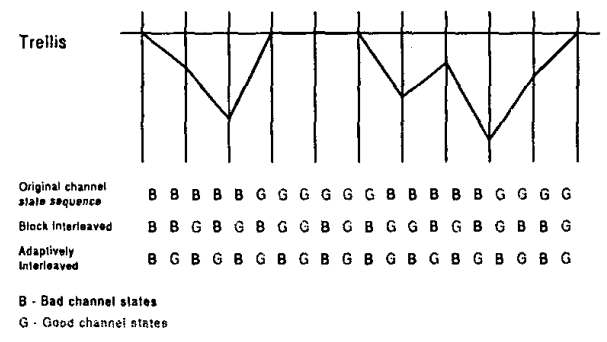

Figure 2: Block interleaving and Adaptive interleaving.

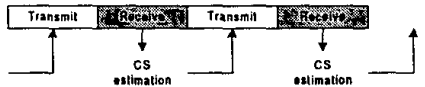

B

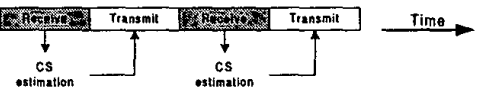

(a) CSI at transmitter in TDD system

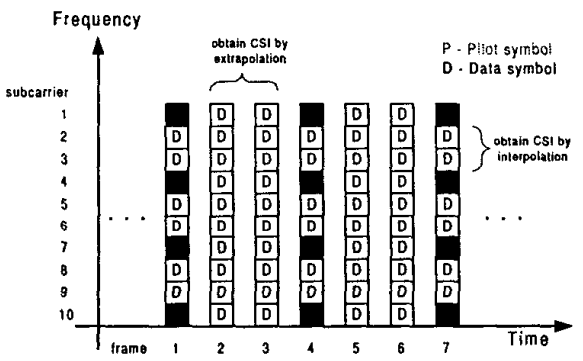

(b) CS estimation by inserting pilot symbols

Figure 3: Channel Estimation
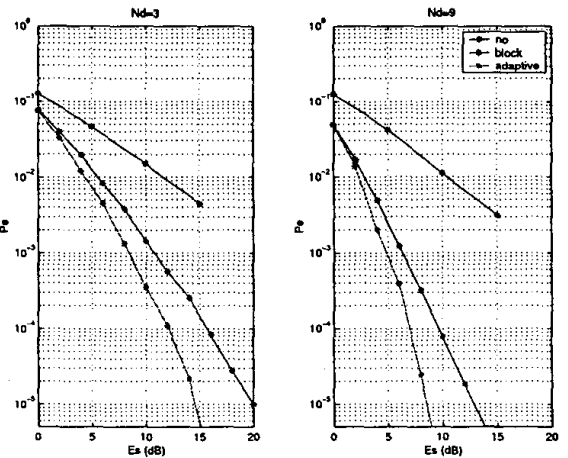

Figure 4: BER for $D_{\min }=5, N_{f}=1024$ 

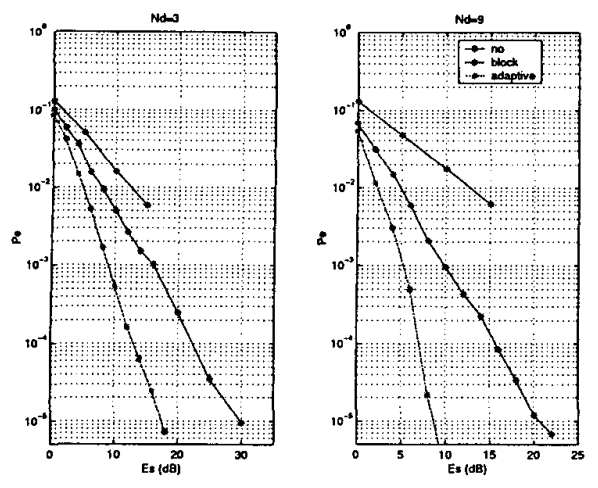

Figure 5: BER for $D_{\min }=5, N_{f}=4096$
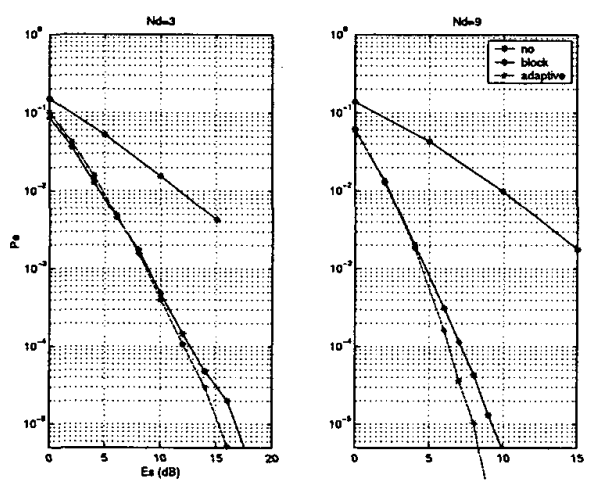

Figure 6: BER for $D_{\min }=7, N_{f}=1024$
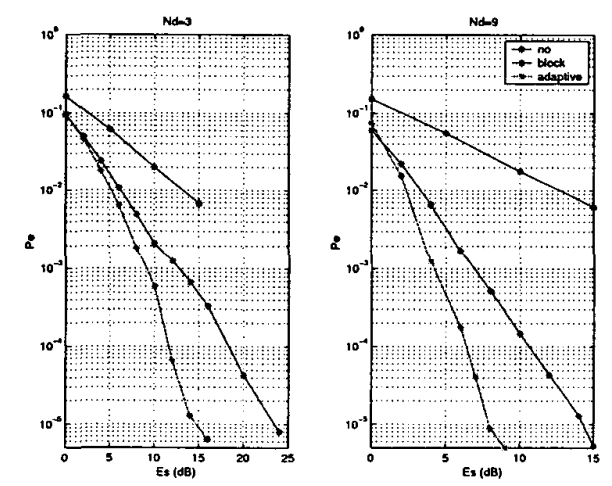

Figure 7: BER for $D_{\min }=7, N_{f}=4096$ 